\title{
Oral hygiene and gingival status in teeth restored with dental amalgam
}

\author{
Vladimir Panov, Martina Markova
}

1. Department of Conservative Dentistry and Oral Pathology, Faculty of Dental Medicine, Medical University, Varna;

\section{Abstract}

Dental amalgam has been a basic restorative material in dentistry for over 150 years due to its low cost, universal availability, ease of application. It has protected many patients from pulp complications, extractions and edentulous. It is still used in many countries, especially in dental care under the universal health insurance system. In addition to environmental problems, this material may have other effects on the surrounding tissue.

We've compared the values of the oral-hygiene and gingival indexes in 43 patients, who have at least one amalgam restoration. Our investigation shows harmful effects of dental amalgam on periodontal health. In most of the parameters we tested, we measured significant differences between teeth reconstructed with dental amalgam and all other teeth. Regarding the Papilla Bleeding Index Ratio the values in all teeth were 0.76 vs 1.33 of these with dental amalgam. We observed also big differences in the Hygiene Index - 52.5\% vs. $80.4 \%$ and in the Debris Index -1 vs. 1.5. We did not report differences only on $\mathrm{OHI}-\mathrm{Cl}$.

We found an extremely high relationship between $\mathrm{DI}$ and $\mathrm{Cl}, \mathrm{DI}$ and $\mathrm{OHI}$. There is also a natural relationship between the higher Oral Hygiene Index (characterized by a larger amount of tartar and plaque) and the result of it - inflammation of the soft tissues of the periodontium (gingivitis), as shown by the Papilla Bleeding Index. Our study shows that teeth restored with dental amalagam can, in addition to having adverse environmental and health effects, causing allergies or increased corrosion potential, contribute to poor periodontal health, maybe due to surface roughness.

Keywords: oral hygiene, gingival inflammation, dental amalgam 


\section{Introduction}

Dental silver amalgam is considered to be one of the most important restorative materials in the history of dental medicine. For more than 200 years, it has been a reliable restorative material. During these years a lot of changes of its composition have been made. Amalgam is still used in many countries. Nearly half of the Bulgarians have at least one amalgam restoration in their mouths (1-3). Dental amalgam is easier to place than other tooth-colored fillings and it improves its marginal tightness over time due to the deposition of corrosion products. Tooth-colored fillings require the area where dentist is working in to be dry. This can be challenging for people with special needs, children and patients who have trouble sitting in the dental chair. Dental amalgam lasts longer than tooth-colored fillings and is also stronger. This strength makes dental amalgam fillings a good choice for restoring molars. Usually amalgam fillings cost less than toothcolored fillings (4).

But dental amalgam contains mercury and can cause reactions in the body or soft tissues. Sometimes patients with amalgam fillings develop increased corrosion potential or allergies $(5,6)$. Amalgam restorations have a rough surface (7). Periodontal health at the restorative gingival interface continues to represent one of the most difficult challenges. The type of restoration, the adaptation of the margins, the contours of the restoration, the proximal contacts and the roughness of the surface have a critical biological impact on the gingiva and the supporting periodontal tissues. Maintenance of a healthy periodontium is substantial for the long-term success of class II and $\mathrm{V}$ dental restorations. These restorations may affect periodontal health, if the distances between the junctional epithelium and supracrestal connective tissue attachment aren't respected, or in the presence of debris due to roughness of the surface, leading to gingival inflammation, connective tissue attachment loss and bone resorption (8-10).

All dental restorative materials should have a highly polished surface to prevent the accumulation of dental plaque and the initiation of periodontal disease. Most restorative materials are biocompatible and are not injurious to periodontal health, with the exception of self-curing acrylics.

But all materials contain surface roughness that can act as foci for plaque accumulation. Restorative resins, over time, have a greater degree of polish attainable but lack in strength and develop porosity. There is also an interaction between the resin and the organic compounds in toothpaste, plaque and soft drinks and there may be softening of the composite material or resin cement leading to surface roughness and plaque retention (10).

A study involving 100 patients with amalgam restoration shows that $51 \%$ had overhanging margins and had shown bone loss (11).

It is agreed that different materials have different molecular distributions and surface chemistry, which could influence bacterial adhesion. This reflects the difference in dental biofilm growth rates on various dental materials (Grivet et al., 2000, Svanberg et al., 1990, Quirynen et al., 1989).

In 2017 Sarra A et al. showed that amalgam induces higher means of plaque, gingivitis and pocket depth compared with composites. The mean of the three parameters, as compared to a control group, were statistically higher in the restored amalgam surfaces $(p<0.05)$. For composite restorations the means of the three parameters were also higher but not significant for both Plaque Index and Gingival Index, except for pocket depth of the restored surfaces were significantly higher than the controlled surfaces. However, there 
were no significant differences in the ranks of the three mesial indices of patients having amalgam in comparison with those having composite restorations. The conclusion of Sarra A et al. suggests that composite restorations did not have a significant advantage on periodontal health over amalgam restorations (12).

If we know the problems, associated with the use of amalgam, we can evaluate better its harmful effects on health and nature.

\section{Aim}

To determine the oral-hygiene and gingival status of teeth restored with dental amalgam and to compare these with those of all of the teeth in the same patients.

\section{Materials and methods}

We've compared the values of the oral-hygiene and gingival indexes in 43 patients who have at least one amalgam restoration.

To evaluate the oral-hygiene status of patients, we chose the Green and Vermillion - OHI Oral Hygiene Index (13), which is a sum of the Debris Index (DI) and the Calculus Index (Cl) and is widely used in dental practice. We reported the index on representative teeth (16, 11, 24, 31 - vestibularly; 36, 46 - lingually). Our goal was to get a quick and accurate idea of the oral-hygiene status of our patients.

The Debris Index (DI) takes into account the distribution of plaque on the teeth surfaces (vestibular and lingual) of the so-called Rumfjord teeth after plaque coloring. The values that we reported were 0, 1, 2, 3.

The Calculus index $(\mathrm{Cl})$ records the distribution of supra- and sub-gingival calculus on the vestibular and lingual surfaces of the Rumfjord teeth. The values wereported were 0 (missing tartar), 1 (supragingival tartar up to $1 / 3$ of the dental surface), 2 (supragingival tartar from $1 / 3$ to $2 / 3$ of the dental surface and / or single findings of subgingival calculus), 3 (supragingival tartar over $2 / 3$ of the tooth surface and / or a ring of subgingival tartar).

We also recorded the Hygiene Index $(\mathrm{HI})$. $\mathrm{HI}$ evaluates the accumulation of dental plaque on the four dental surfaces. Working with this index, the plaque should be colored. The presence of plaque is noted with " + " and the absence with " - ". The plaque-free surfaces should be calculated in percentage (the number of plaque-free surfaces: the number of surfaces tested) $\times 100=\%$. Values of 0 correspond to excellent hygiene; values from 0.1 to 2.4 to good hygiene; values from 2.5 to 6.0 correspond to satisfactory hygiene and values from 6.1 to 12.0 are a proof of poor hygiene.

To assess gingival inflammation we used the Papilla Bleeding Index of Saxer and Muhleman (PBI) (14).

The probing was performed orally in the first and third quadrant and vestibularly - in the second and fourth quadrant. Papilla Bleeding Index was calculated by dividing the sum of the index numbers by the number of papillae examined. A value of 0 corresponds to lack of bleeding, 1 - to a single point of blood, 2 - to 
several points or a thin line of blood, 3 - to an interdental triangle filled with blood, and the highest value of 4 - corresponded to profuse bleeding.

The bleeding after gingival stimulation (the so-called provoked bleeding) is considered as a reliable sign of gingival inflammation.

\section{Results}

We've compared the values of 43 patients in terms of indices, using oral-hygiene and gingival status. Part of the results are shown in Table 1.

Table 1. Results from oral hygiene and gingival indices.

\begin{tabular}{|l|l|l|l|l|l|l|}
\hline Number of amalgam restorations & DI & CI & DI+CI & PBI & PBI-amalgam & HI \% \\
\hline 2,00 & 6 & 4 & 10,00 & 0,80 & 2,00 & 46 \\
\hline 7,00 & 14 & 13 & 27,00 & 2,70 & 3,00 & 26 \\
\hline 4,00 & 2 & 0 & 2,00 & 0,44 & 1,00 & 64 \\
\hline 6,00 & 5 & 1 & 6,00 & 0,44 & 1,67 & 52 \\
\hline 4,00 & 10 & 6 & 16,00 & 2,20 & 2,50 & 49 \\
\hline 1,00 & 3 & 0 & 3,00 & 1,03 & 1,00 & 57 \\
\hline
\end{tabular}

The Hygiene Index estimates the accumulation of dental plaque on the four tooth surfaces. On teeth restored with amalgam, we reported an average Hygiene Index of $80.4 \%$, and for all of the same patient's teeth, we recorded a value of $52.54 \%$ (Fig. 1).

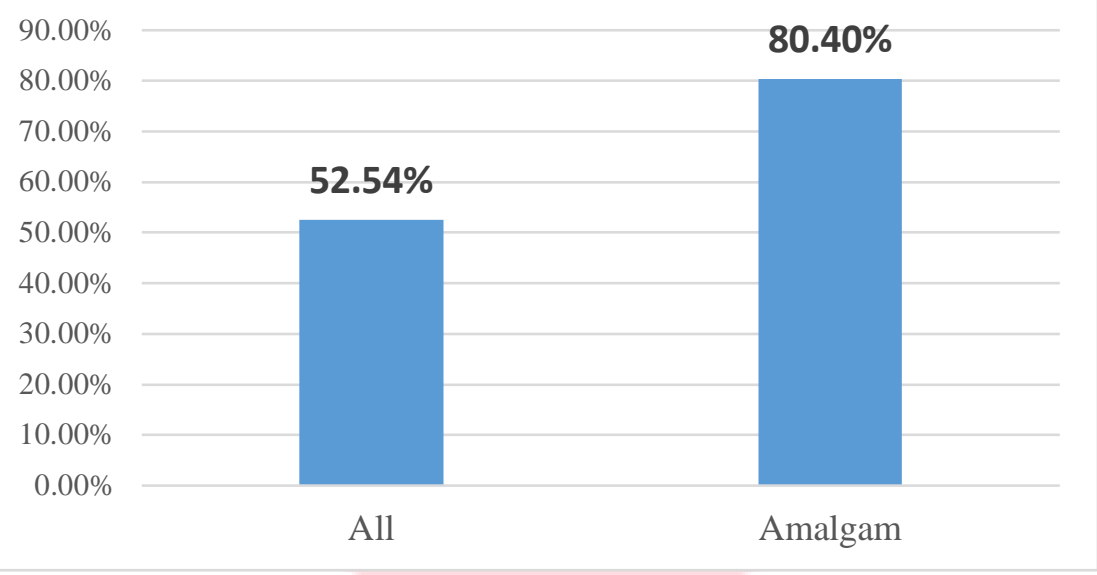

Figure 1. HI ratio in all teeth and those with dental amalgam. 
Our research shows undisputed accumulation of greater amount of plaque on teeth restored with dental amalgam, which is a prerequisite for the development of carious lesions and periodontal diseases. The differences that we found are very significant. In more than $73 \%$ of the cases, teeth with amalgam restorations had a higher Hygiene Index than the average value for the same patient.

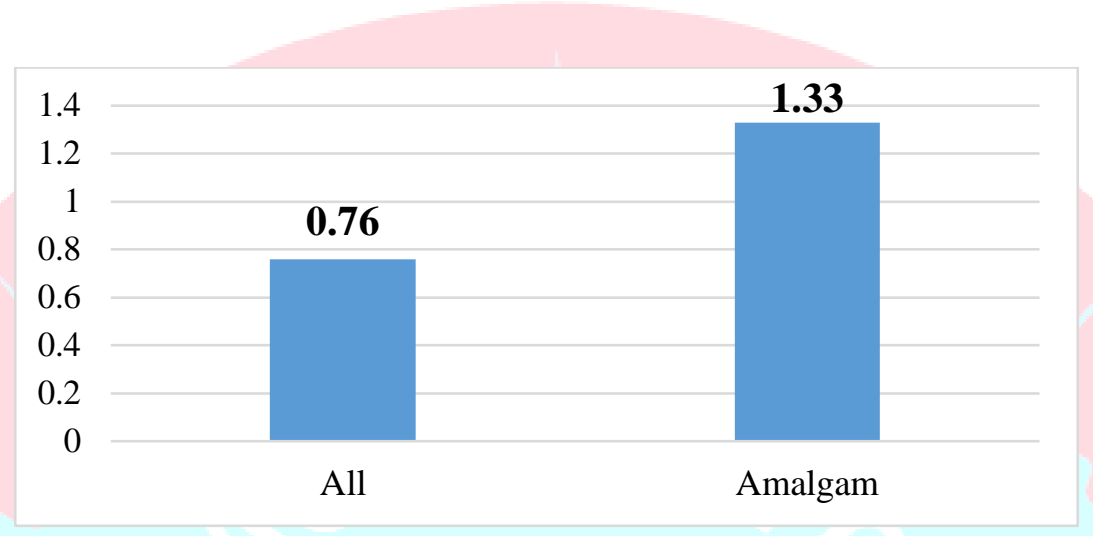

Figure 2. PBI ratio in all teeth and those restored with dental amalgam.

We found almost twice higher PBI-values in teeth with dental amalgam - 1.33 (Rank 0.66-3; SD 0.54) compared to all teeth - 0.76 (Rank 0.25-2.7; SD 0.58) (Fig. 2). This shows a much stronger gum inflammation around teeth restored with amalgam.

Regarding OHI-DI-index, we found that it was much higher in teeth with dental amalgam compared to all the surfaces reported by us (Fig. 3)

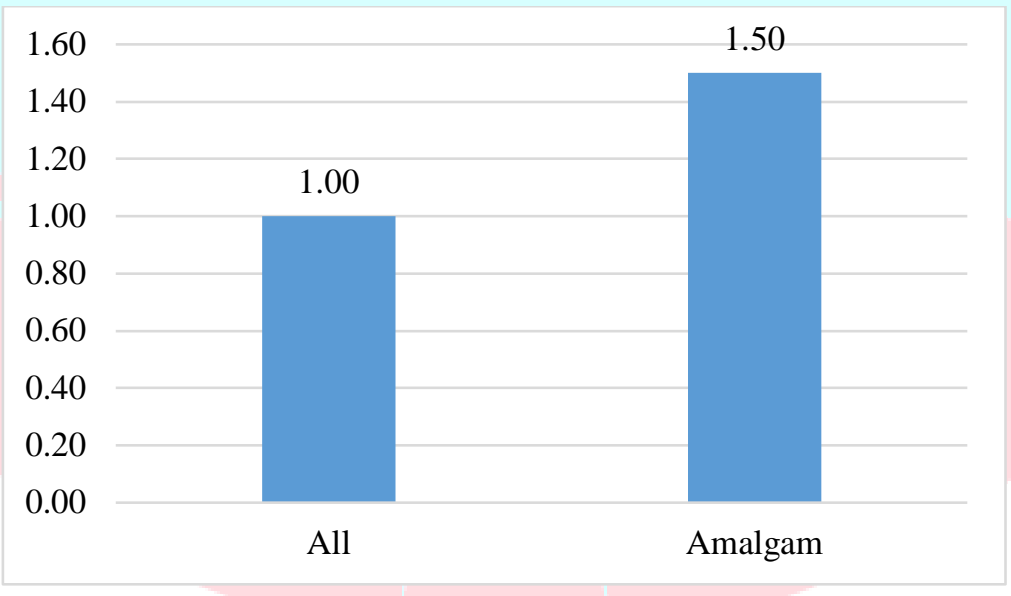

Figure 3. OHI-DI ratio in all teeth and those with dental amalgam.

We did not report differences on $\mathrm{OHI}-\mathrm{Cl}$. 
Table. 2 Pearson correlations of the studied parameters.

\begin{tabular}{|c|c|c|c|c|c|c|}
\hline & DI & CI & OHI & PBI-all & $\begin{array}{l}\text { PBI- } \\
\text { Amalgam }\end{array}$ & HI \\
\hline \multirow[t]{2}{*}{ DI } & 1 & ,616 &, $893^{* *}$ &.$^{\mathrm{a}}$ & 135 &,$- 580^{* *}$ \\
\hline & & ,001 & ,000 & . & ,501 & ,002 \\
\hline \multirow[t]{2}{*}{ CI } & ,616 & 1 &, $905^{* *}$ &.$^{a}$ &, $586^{* *}$ &,$- 392^{*}$ \\
\hline & ,001 & &, 000 & e & ,001 & ,043 \\
\hline \multirow[t]{2}{*}{$\mathrm{OHI}$} & $893^{* *}$ &, $905^{* *}$ & 1 &.$^{\mathrm{a}}$ &, $408^{*}$ &,$- 538^{* *}$ \\
\hline & ,000 & ,000 & & . & 035 & ,004 \\
\hline \multirow[t]{2}{*}{ PBIall } &.$^{\mathrm{a}}$ &.$^{\mathrm{a}}$ &.$^{\mathrm{a}}$ &.$^{\mathrm{a}}$ &.$^{\mathrm{a}}$ &.$^{a}$ \\
\hline & . & . & . & & . & . \\
\hline \multirow{2}{*}{$\begin{array}{l}\text { PBIAmalg } \\
\text { am }\end{array}$} & ,135 &, $586^{* *}$ & ,408* &.$^{a}$ & 1 & ,006 \\
\hline & ,501 & ,001 & ,035 & e & & ,976 \\
\hline \multirow[t]{2}{*}{ HI } &,$- 580^{* *}$ &,$- 392^{*}$ &,$- 538^{* *}$ &.$^{\mathrm{a}}$ & ,006 & 1 \\
\hline & ,002 & ,043 & ,004 & . & ,976 & \\
\hline
\end{tabular}

* The correlation is significant at the 0.05 level; **The correlation is significant at the 0.01 level.

Numerous correlation relationships were found in Pearson analysis (Table 2). We found an extremely high relationship between $\mathrm{DI}$ and $\mathrm{Cl}, \mathrm{DI}$ and $\mathrm{OHI}$. There is also a natural relationship between the higher Oral Hygiene Index (characterized by a larger amount of tartar and plaque) and the result of it - inflammation of the soft tissues of the periodontium (gingivitis), as shown by the Papilla Bleeding Index.

\section{Conclusion}

Our investigation shows harmful effects of dental amalgam on periodontal health. In most of the parameters we tested, we measured significant differences between teeth reconstructed with dental amalgam and all other teeth. Regarding Papilla Bleeding Index Ratio the values in all teeth were 0.76 vs. 1.33 of these with dental amalgam. We observed also big differences in the Hygiene Index $-52.5 \%$ vs. $80.4 \%$ and in the Debris Index -1 vs. 1.5. We did not report differences only on $\mathrm{OHI}-\mathrm{Cl}$.

We know that the presence of dental plaque can cause a lot of complications such as gingivitis-like inflammatory lesions, mediated by host-parasite interactions; periodontitis which leads to bone destruction; diseases linked to periodontitis; and dental caries by demineralization of the enamel, which can progress to further breakdown of the more organic, inner dental tissue with different tissue involvement. Most likely, increased plaque retention is due to the increased roughness of the dental amalgam obturations. 
Our study shows that teeth restored with dental amalagam can, in addition to having adverse environmental and health effects, or causing allergies or displaying increased corrosion potential, contribute to poor periodontal health.

\section{References}

1. Markova M, Panov VI, Georgiev G, Vicheva G, Dental amalgam - clinical and epidemiological aspects, Varna Medical Forum, vol. 5, 2016, issue 2, 210-213.

2. Panov $\mathrm{Vl}$, Side effects in the mouth when using dental amalgam, Varna Medical Forum, vol. 5, 2016, issue 1, 77-81.

3. Panov VI, M. Markova, History of Dental Amalgam, Varna Medical Forum, vol. 5, 2016, issue 1, 119-123.

4. Rho, Y.J., Namgung, C., Jin, B.H., Lim, B.S., Cho, B.H. Longevity of direct restorations in stressbearing posterior cavities: a retrospective study. - Oper Dent., 2013, 38(6):572-582.

5. Balcheva M, Panov VI, Krasteva A, Markova M. Allergy to dental amalgam, Medinform, 6(1), 2019, 968-974.

6. Panov VI, Markova M, Corrosion potential in oral cavity, Medinform, 2018;5(1), 750-759.

7. P V Raju, B R Varma, K M Bhat, Periodontal Implications of Class II Restorations. Clinical and SEM Evaluation, Indian J Dent Res, 1996, 7 (1), 21-27.

8. AL-Sanjary S, Gasgoos S, Effect of Class II Amalgam and Composite Restorations on Periodontal Health of Posterior Teeth: An in vivo study, JODR, Volume 4, Issue 2, 2017, (8292).

9. Rasines Alcaraz MG, Veitz-Keenan A, Sahrmann P, et al. Direct composite resin fillings versus amalgam fillings for permanent of adult posterior teeth. Cochrane Database Syst Rev 2014:CD005620.

10. Yasser Al-Fawaz, Raneem Alofi, Hafez Diab. Comparative study between the effect of class II amalgam and composite restorations in posterior teeth on periodontal tissues health. E.D.J. Vol. 63, No. 3, (1-7).

11. Ramamurthy J, Effect of Restorations on Periodontal health, IOSR Journal of Dental and Medical Sciences, 2014, 13(7), 71-73.

12. Sarra A. AL-Sanjary, Saher S. Gasgoos. Effect of Class II Amalgam and Composite Restorations on Periodontal Health of Posterior Teeth: An in vivo study, JODR, 2017. 4(2), 82-92.

13. Greene, J.C., Vermillion, J.R. The simplified oral hygiene index. - J. Am. Dent. Assoc., Jan, 1964; 68: 7-13.

14. Saxer, U.P., Mühlemann, H.R. Motivation and education. SSO Schweiz Monatsschr Zahnheilkd., Sep, 1975, 85(9):905-919.

\section{Corresponding author:}

Vladimir Panov, DMD, PhD, DSc,

Professor, Head of Department of Conservative Dentistry,

and Oral Pathology, Faculty of Dental Medicine,

Medical University, Varna,

email: vladimir.panov@mu-varna.bg 